Nicola Wagner ${ }^{1}$

C. Rose $^{2}$

Eva-B. Bröcker ${ }^{2}$
Osteoma cutis

\section{Osteoma Cutis}

\section{Zusammenfassung}

Bei einem 66-jährigen Mann traten seit 10 - 15 Jahren primäre Osteome der Haut auf. Primäre Knochenneubildungen in der Haut entstehen selten und unabhängig von präexistenten Hautveränderungen oder vorausgegangenen Traumata. Eine Osteombildung im Rahmen des Albright- oder Gardner-Syndroms sollte ausgeschlossen werden. Davon unterschieden werden die unabhängigen primären Osteome, die isoliert oder generalisiert oder multipel als miliare Osteome des Gesichtes auftreten. Schließlich sollte eine sekundäre Osteombildung bei inflammatorischen Erkrankungen, in Tumoren oder posttraumatischer Genese abgegrenzt werden. Therapeutisch sind sowohl die Exzision als auch die operative Entfernung mithilfe des Erbium-Yag:Lasers möglich.

\section{Abstract}

Multiple cutaneous osteomas occured in a 66-year old man since 10-15 years. Primary bone formation in the skin occurs rarely and independently from preexisting skin lesions or preceding trauma. An incidence of osteoma in association to Albright's or Gardner's syndrome should be excluded. Osteoma cutis can be found isolated or generalized as well as multiple miliary osteoma of the face. Finally, cutaneous bone formation secondary to inflammatory diseases, neoplasia or trauma has to be separated from the primary forms. Excision or removal by Erbium-Yag-Laser are treatment modalities.

\section{Einleitung}

Primäre Osteome der Haut sind ein seltenes Krankheitsbild. Sie entstehen unabhängig von präexistenten Hautveränderungen und zeigen histologisch eine Knochenneubildung in der Haut. Das Alter der Patienten bei Entstehung, die Abgrenzung von sekundären Osteomen und Kalzifikationen stellen für Prognose und Therapie wesentliche Kriterien dar.

\section{Anamnese}

Bei einem 66-jährigen Mann waren seit 10-15 Jahren langsam zunehmend, derbe Papeln an der Brust aufgetreten, die ihn wegen des Juckreizes zu einem Hautarzt geführt hatten. Es war weder ein Trauma noch eine Akne vorangegangen. Es bestanden keinerlei Grunderkrankungen. Medikamente nahm der Patient nicht regelmäßig ein. 
Abb. 1 Multiple hautfarbene derbe Papeln am Dekolletee.
Dermatologischer Befund (Abb.1 u. 2)

Im gesamten Brustbereich, betont sternal, bestehen multiple rötliche bis hautfarbene, teils ulzerierte sehr derbe Papeln bis zu $7 \mathrm{~mm}$ Größe. Druckdolenz bestand nicht. Eine auffällige Physiognomie oder Skelettveränderungen lagen nicht vor.

\section{3-MHz-Sonographie (Abb. 3)}

Multiple bis zu $7 \mathrm{~mm}$ durchmessende, scharf begrenzte echoreiche Raumforderungen in der Dermis mit deutlichen Schallschatten.

\section{Histopathologie (Abb. 4)}

In der retikulären Dermis findet sich scharf begrenzt regulär differenziertes Knochengewebe. Im Markraum des spongiösen Lamellenknochen sieht man reifzelliges Fettgewebe und Blutgefäße. Entzündliche Infiltrate und Narbengewebe fehlen. Die Epidermis und die Adnexe sind normal strukturiert. Beurteilung: kutanes Osteom.

Labor

Normwerte für Differenzialblutbild, Kalzium, Leber- und Nierenwerte, anorganisches Phosphat, Parathormon, FT3, FT4, TSH. Unauffällige Kalziumausscheidung im 24 h-Urin.

\section{Röntgenaufnahme des Thorax}

Unauffällig.

Koloskopie

Unauffällig.

Abb. 3 Echoreicher Plaque in der Dermis mit ausgeprägtem Schallschatten.

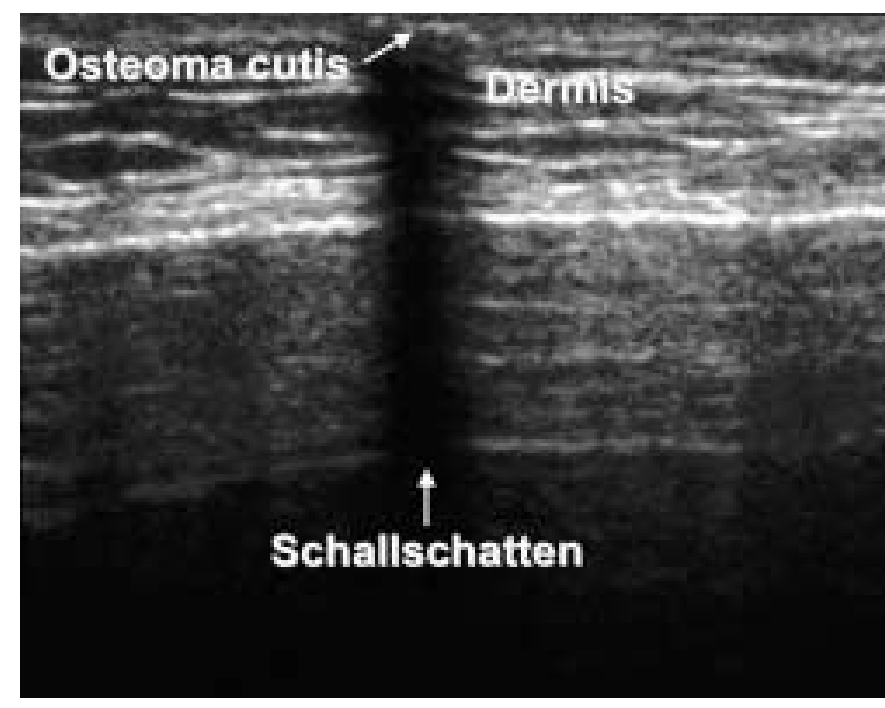

Abb. 4 Histologie eines Osteoms. In der Dermis gelegenes, gut umschriebenes Knochengewebe (Hämatoxylin und Eosin-Färbung). 
Im geschilderten Fall liegen primäre kutane Osteome vor [3]. Es ergab sich kein Anhalt für ein Albright- oder Gardner-Syndrom. Pathologische Laborveränderungen, andere Neoplasien oder vorangegangene Traumata bzw. eine stattgehabte Akne vulgaris bestanden nicht. Eine bildgebende Darstellung der kutanen Osteome gelingt mit der $13 \mathrm{MHz}$-Sonographie, so dass die früher gebräuchlichen, belastenden Röntgenweichstrahlenaufnahmen nicht mehr notwendig sind.

Unsere Fallbeschreibung gleicht den Berichten von Goldminz [7] und Gfesser [5], deren Patienten ebenfalls keine zusätzlichen Osteome im Gesicht aufwiesen. Dies mag ein Indiz dafür sein, dass kutane Osteome auch unabhängig von einer vorangegangenen Akne vulgaris entstehen können.

Die Pathogenese kutaner Osteome ist nicht geklärt. Möglicherweise persistieren pluripotente mesenchymale Zellen, aus denen Osteoblasten entstehen, oder gewebsständige Fibroblasten differenzieren zu Osteoblasten [9].

Die kutane Ossifikation wird in primäre und sekundäre Osteome unterteilt (Tab.1). Primäre Osteome sind ein Symptom der hereditären Osteodystrophie Albright. Sie können bei Geburt vorhanden sein oder im Laufe des Lebens entstehen. Werden Osteome im Kindesalter diagnostiziert, sollte ein Albright-Syndrom ausgeschlossen werden [6]. Neben Kleinwuchs, Skelettanomalien, Katarakt, kurzen und breiten Nägeln, Oligophrenie, großflächige Café-au-lait Pigmentierungen und Osteoporose kann auch eine Hyperphosphatämie und Hypokalzämie als Folge eines Pseudohypoparathyreoidismus (Endorganresistenz des Parathormons) entstehen [6]. Der teilweise beim Albright-Syndrom vorkommende Pseudopseudo-Hypoparathyreoidismus zeichnet sich durch normale Serumwerte für Kalzium und Phosphat aus.

Normwertige Kalzium- und Phosphatspiegel finden sich bei allen weiteren primären Osteomen der Haut. Das Gardner-Syndrom (familiäre Polypose des Dickdarms) geht mit einer nahezu $100 \%$ igen Inzidenz von Kolonkarzinomen einher. Weitere Symptome können Epidermalzysten, Lipome und Osteome sein, wobei letztere meist im Kiefer- oder Schädelbereich lokalisiert sind und häufig bereits im Kindesalter auffallen [5].

Tab. 1 Diffenzialdiagnosen kutaner Osteome

\begin{tabular}{|c|c|}
\hline primäre Osteome & sekundäre Osteome \\
\hline assoziiert mit Albright-Syndrom & 1. bei entzündlichen Erkrankungen \\
\hline assoziiert mit Gardner-Syndrom & 2. in Tumoren/Neoplasien \\
\hline unabhängige Osteome: & 3. posttraumatisch, in Narben \\
\hline \multicolumn{2}{|l|}{$\begin{array}{l}\text { a) multiple miliare Osteome des } \\
\text { Gesichtes }\end{array}$} \\
\hline \multicolumn{2}{|l|}{ b) isolierte Osteome } \\
\hline \multicolumn{2}{|l|}{ c) generalisierte Osteome } \\
\hline \multicolumn{2}{|l|}{ - kongenitale plaque-artige Osteome } \\
\hline - progressive ossäre Heteroplasie & \\
\hline
\end{tabular}

Multiple miliare Osteome treten vorrangig bei Frauen mittleren Alters im Gesicht auf. Da anamnestisch oft eine Akne zu eruieren ist, bleibt unklar, ob es sich wirklich um primäre oder nicht eher um sekundäre Osteome handelt. In Einzelfällen erscheinen diese Osteome bläulich, was durch eine vorausgegangene Tetrazyklinoder Minozyklintherapie erklärt wird. Das Tetrazyklin wird in das Knochengewebe eingebaut und führt zu einer Braunverfärbung des Knochens. Da tief gelegenes braunes Pigment langwelliges Licht absorbiert und kurzwelliges Licht reflektiert, resultiert der klinisch blaue Farbton. Aufgrund der Geschlechtsverteilung wurde Östrogen als Stimulator der Osteoblasten diskutiert. Bisher blieb die Beweisführung jedoch aus [1].

Isolierte Osteome können als harter Nodus an jeglicher Körperstelle entstehen. Die häufige Lokalisation am Kopf lässt an eine ursächliche Versprengung embryonalen Knochengewebes denken. Oft manifestieren diese sich erst bei älteren Kindern oder Erwachsenen $[4,14]$.

Generalisierte Osteome beobachtet man in der Neonatalperiode [17]. Die ebenfalls in der Neonatalzeit auftretenden plaque-artigen Osteome weisen eine differente Prognose auf und lassen sich in zwei Gruppen aufteilen [10]: eine gute Prognose haben große subkutane ossäre Plaques an den Extremitäten oder dem Kapillitium, die einen chronisch stabilen Verlauf zeigen. Im Gegensatz dazu zeigt die „progressive ossäre Heteroplasie“ (POH), die erstmals 1994 in der orthopädischen Literatur beschrieben wurde [8], eine zunehmende heterotope Ossifikation der Haut und der Weichteile. Der Beginn kann in die ersten Lebensmonate fallen. Protopathisch beginnt die Ossifikation in der Dermis und kann Fett- und Muskelgewebe involvieren. Eine Hautbeteiligung wird oft durch reiskornartige Papeln apparent. Resultierende Ankylosen oder Beinlängendifferenzen können eine orthopädische Intervention erforderlich machen. Alkalische Phophatase, Kreatinkinase und Laktatdehydrogenase sind fakultativ erhöht.

Sekundäre Osteome werden im Rahmen entzündlicher Erkrankungen wie Syphilis, Sklerodermie, Dermatomyositis und posttraumatisch beschrieben. Berichtet wird auch über die Entste-

Tab. 2 Differenzialdiagnosen kutaner Kalzifikationen

\begin{tabular}{|c|c|c|c|}
\hline dystrophisch & metastatisch & idiopathisch & iatrogen \\
\hline kutane Neoplasmen & $\begin{array}{l}\text { primärer Hyper- } \\
\text { parathyreoidismus }\end{array}$ & $\begin{array}{l}\text { kutane } \\
\text { Neoplasien }\end{array}$ & $\begin{array}{l}\text { i.v. Ca- } \\
\text { Gluconat }\end{array}$ \\
\hline Kollagenosen & $\begin{array}{l}\text { chronische Nieren- } \\
\text { insuffizienz }\end{array}$ & $\begin{array}{l}\text { skrotale Kalzi- } \\
\text { fikationen }\end{array}$ & \\
\hline $\begin{array}{l}\text { Prophyria cutanea } \\
\text { tarda }\end{array}$ & Kalziphylaxie & & \\
\hline Werner-Syndrom & Sarkoidose & & \\
\hline $\begin{array}{l}\text { Ehlers-Danlos- } \\
\text { Syndrom }\end{array}$ & Hypervitaminose D & & \\
\hline Trauma & Neoplasmen & & \\
\hline \multicolumn{4}{|l|}{ Infektionen } \\
\hline \multicolumn{4}{|l|}{ Pannikulitis } \\
\hline \multicolumn{4}{|l|}{ Infektionen } \\
\hline $\begin{array}{l}\text { Pseudoxanthoma } \\
\text { elasticum }\end{array}$ & & & \\
\hline
\end{tabular}


hung sekundärer Osteome in Pilomatrikomen, melanozytären Naevi, Basaliomen, Adnextumoren, Histiozytomen, Hämangiomen, Fibroxanthomen und bei chronisch venöser Insuffizienz $[10,14]$.

Die Osteombildung ist abzugrenzen von der Kalzifikation, d.h. dermalen oder subkutanen Kalziumdepots ohne Anzeichen von Verknöcherung. Man unterscheidet metastatische, dystrophische, idiopathische und iatrogene Kalzifikationen (Tab. 2).

Die so genannte metastatische Kalzifikation der Haut resultiert aus einem Ungleichgewicht des Kalzium- und Phosphathaushaltes. Häufig liegt eine Multi-Organbeteiligung vor, insbesondere der Nieren, Lungen und Gefäße. Kausal wird die geringe Phosphatausscheidung bei Niereninsuffienz angenommen. Typischerweise sind derartige Kalzifikationen periartikulär verteilt und lösen sich bei Normalisierung der Kalzium- und Phosphatwerte wieder auf [4]. Eine ernste Prognose hat die metastatische Kalziphylaxie, die bei Langzeit-Dialysierten und HIV-Patienten mit Niereninsuffizienz auftritt. Klinisch zeigen sich, aufgrund extensiver Kalzifikationen der kleineren und mittleren Gefäße, ausgedehnte schmerzhafte Livedo-reticularis-artige Hautveränderungen, die in flächige Ulzerationen übergehen können. Ursächlich ist die Erhöhung des Kalzium-Phosphatproduktes in Kombination mit einem prädisponierenden Faktor, dem Parathormon, das die Kalziumablagerung im Gewebe erleichtert, verantwortlich [2]. Therapeutisch ist eine Parathyreoidektomie mit Autotransplantation einer Drüse in einen Muskel vorzunehmen. Diese kann bei ausbleibender Besserung zusätzlich exstirpiert werden.

So genannte dystrophe Kalzifikationen manifestieren sich lokal ohne eine generalisierte Störung des Kalzium/Phosphatgleichgewichtes. Häufig werden sie bei Dermatomyositis und systemischer Sklerodermie, insbesondere dem Crest-Syndrom, beobachtet. Diverse Infektionen und entzündliche Veränderungen der Haut können ebenfalls von Kalzifikationen begleitet sein (s. Tab. 2).

Kalzifikationen bei juveniler Dermatomyositis und systemischer Sklerodermie sprechen therapeutisch teilweise auf Diltiazem an [13]. Als Wirkmechanismus wurde postuliert, dass durch Diltiazem der Kalzium-Influx in das Gewebe verhindert wird und Makrophagen bestehende Kalzifikationen in der Folge abbauen können.

Für kutane Osteome bieten sich folgende Therapien an: kleinere, gut erreichbare Osteome können exzidiert oder durch Punch- biopsien ggf. auch durch Dermabrasion entfernt werden [7]. Gute kosmetische Ergebnisse werden bei oberflächlich liegenden Osteomen durch die Erbium:YAG-Laser assistierte Abtragung der Gewebsschichten, die über den Osteomen liegen, und anschließender Kürettage bzw. Exstirpation erzielt $[11,12,16]$. Bei multiplen miliaren Osteomen ist auch eine Therapie mit 0,025\% Tretinoingel über mehrere Monate versucht worden. [15]. Die orale Applikation von Editronsäure, einem synthetischen Hemmstoff des Knochenumbaus, erwies sich als enttäuschend $[7,13]$. Unser Patient fühlte sich durch die multiplen dermalen Osteome kosmetisch kaum beeinträchtigt und wünschte bisher keine Therapie.

\section{Literatur}

${ }^{1}$ Boehncke WH, Kaufmann R, Weber L, Sterry W. Osteoma cutis. Hautarzt 1993; 44: 245-247

${ }^{2}$ Cockerell CJ, Dolan E. Widespread cutaneous and systemic calification (calciphylaxis) in patients with the acquired immunodeficiency syndrome and renal disease. J Am Acad Dermatol 1992; 26: 559-562

${ }^{3}$ Cottoni F, DelíOrbo C, Quacci D, Tedde G. Primary osteoma cutis: clinical, morphological and ultrastructural study. Am J Dermatopathol 1993; 15: 77-81

${ }^{4}$ Fazeli P, Harvell J, Jacobs MB. Osteoma cutis (cutaneous ossification). West J Med 1999; 171 (4): 243-245

${ }^{5}$ Gfesser M, Worret WI, Hein R, Ring J. Multiple primary osteoma cutis. Arch Dermatol 1998; 134 (59): 641 -3

${ }^{6}$ Goeteyn V, De Potter CR, Naeyaert JM. Osteoma cutis in pseudohypoparathyreoidism. Dermatology 1999; 198 (2): 209-11

7 Goldminz D, Greenberg R. Multiple miliary osteoma cutis. J Am Acad Dermatol 1991; 24: $878-881$

${ }^{8}$ Kaplan FS, Craver R, Mac Ewan GD. Progressive osseous heteroplasia: a distinct developmental disorder of heterotopic ossification. J Bone Joint Surg Am 1994; 76 A : 425-436

9 Mast AM, Hansen R. Multiple papules on the elbow: congenital osteoma cutis. Arch Dermatol 1997; 133: 775 - 780

${ }^{10}$ Miller ES, Esterly NB, Fairley JA. Progressive osseous heteroplasia. Arch Dermatol 132 1996; (7): 787-91

${ }^{11}$ Ochsendorf FR, Kaufmann R. Erbium:YAG laser-assisted treatment of miliary osteoma cutis. Br J Dermatol 1998; 138 (2): 371 - 72

12 Ochsendorf FR, Kaufmann R. Erbium:YAG. laser ablation of osteoma cutis: modifications of the approach. Arch Dermatol 1999; 135 (11): 1416

${ }^{13}$ Oliveri MB, Palermo R, Mautalen C, Hubscher O. Regression of calcinosis during diltiazem treatment in juvenile dermatomyositis. J Rheumatol 1996; 23: $2152-2155$

${ }^{14}$ Schumachers G, Worret WI. Osteoma cutis. Hautarzt 1992; 43: $422-425$

${ }^{15}$ Smith CG, Glaser DA. Treatment of multiple miliary osteoma cutis with tretinoin gel. J Am Acad Dermatol 1999; 41: 500

${ }^{16}$ Stöckel S, Eppinger S, Stein A, Meurer M. Multiple miliare Osteome des Gesichts. Hautarzt 2002; 53: 37-41

${ }^{17}$ Walsh JS, Fairley J. Calcifying disorders of the skin. J Am Acad Dermatol 1995; 33: $693-706$ 